\title{
CONTINUED FRACTIONS AND CROSS-RATIO GROUPS OF CREMONA TRANSFORMATIONS*
}

BY H. S. WALL

1. Introduction. An arbitrary cross ratio

$$
r_{i j k l}=\left[z_{i}, z_{j}, z_{k}, z_{l}\right]
$$

of four of the independent variables $z_{1}, z_{2}, \cdots, z_{n}$ is expressible rationally in terms of the ratios of any fundamental system such as

C:

$$
s_{i}=\left[z_{i-1}, z_{i-2}, z_{i}, z_{i-3}\right], \quad(i=4,5, \cdots, n),
$$

or

$M:$

$$
r_{i}=\left[z_{1}, z_{2}, z_{3}, z_{i}\right],
$$$$
(i=4,5, \cdots, n),
$$

of $n-3$ independent ratios. If any particular system of $n-3$ independent ratios be associated with a particular order of the variables by varying the order of the $n$ variables, we shall have in all $n$ ! conjugate systems; these systems are expressible rationally in terms of the original system and in terms of any system of the set. Hence arises a group of $n$ ! Cremona transformations on $n-3$ variables. E. H. Moore, $\nmid$ H. E. Slaught $\ddagger$ and others have studied the group based on the initial system $M$.

In this paper I have shown that the transformations based on the system $C$ have application to continued fractions of the form

$$
\xi=1-\frac{x_{1}}{1}-\frac{x_{2}}{1}-\frac{x_{3}}{1}-\ldots
$$

If we take for the $n$ variables $z_{1}, z_{2}, \cdots, z_{n}$ any $n$ consecutive convergents of the continued fraction, say

$$
z_{i}=\frac{A_{q+i}}{B_{q+i}}, \quad(i=1,2, \cdots, n ; q \geqq-1),
$$

* Presented to the Society, under a somewhat different title, April 6, 1934. $\dagger$ E. H. Moore, American Journal of Mathematics, vol. 22 (1900), pp. 279291.

$\ddagger$ H. E. Slaught, ibid., pp. 343-380, and Part II in vol. 23. 
then in this case the fundamental system $C$ is $n-3$ consecutive elements $x_{i}$ of the continued fraction $\xi$, namely,

$$
s_{i}=x_{q+i}, \quad(i=4,5, \cdots, n) .
$$

The Cremona transformations are on these elements, and their effect is to permute $n$ consecutive convergents of $\xi$ among themselves. The transformations do not disturb the convergence or divergence of the continued fraction, and result therefore in new convergence criteria.

2. The Group $C_{n !}$ of Cremona Transformations. Let $A_{m, t} / B_{m, t}$, ( $t \geqq 0, A_{m, 0} / B_{m, 0}=A_{m} / B_{m}$ ), be the $m$ th convergent of the continued fraction

$$
1-\frac{x_{1+t}}{1}-\frac{x_{2+t}}{1}-\frac{x_{3+t}}{1}-\ldots
$$

Then with the aid of the identity*

$$
A_{n+t-1} B_{t-1}-A_{t-1} B_{n+t-1}=-x_{1} x_{2} \cdots x_{t} B_{n-1, t},
$$

we find that a cross ratio $r_{i j k l}=\left[z_{i}, z_{j}, z_{k}, z_{l}\right],(i<j<k<l)$, of four of the $n$ variables (1) can be expressed in the form

$$
r_{i j k l}=\frac{B_{k-i-1, q+i+1} B_{l-j-1, q+j+1}}{B_{k-j-1, q+j+1} B_{l-i-1, q+i+1}} .
$$

The other five distinct ratios of these four variables can be obtained from the ratio (3) by the well known transformations

$$
r_{j i k l}=\frac{1}{\lambda}, \quad r_{i k j l}=1-\lambda, \quad r_{k i j l}=\frac{1}{1-\lambda},
$$

$$
r_{k j i l}=\frac{\lambda}{\lambda-1}, \quad r_{j k i l}=\frac{\lambda-1}{\lambda},
$$

where $\lambda=r_{i j k l}$. In particular, we find that

$$
s_{i}=x_{q+i}, \quad(i=4,5, \cdots, n) .
$$

Inasmuch as (3) depends upon $x_{q+4}, x_{q+5}, \cdots, x_{q+n}$ only, it follows that (4) is a fundamental system of cross ratios.

Let $j_{1}, j_{2}, \cdots, j_{n}$ be an arbitrary permutation of $1,2, \cdots, n$; and put

* Perron, Die Lehre von den Kettenbrïchen, 1st ed., p. 17. 
1934.]

$$
a=\left(\begin{array}{ccc}
1, & 2, \cdots, & n \\
j_{1}, & i_{2}, \cdots, & i_{n}
\end{array}\right)
$$

Then

$C^{a}$ :

$$
s_{i}^{a}=\left[z_{j_{i-1}}, z_{j_{i-2}}, z_{j_{i}}, z_{j_{i-3}}\right], \quad(i=4,5, \cdots, n),
$$

is also a fundamental system; and $C^{a}$ is expressible rationally in terms of $C$ :

$$
s_{i}^{a}=f_{i}^{a}\left(s_{4}, s_{5}, \cdots, s_{n}\right), \quad(i=4,5, \cdots, n),
$$

or, simply, $C^{a}=f^{a} C$.

If $b$ is another permutation, then $C^{a b}=f^{a} C^{b}=f^{a b} C$. The $n$ ! Cremona transformations $f^{a}, f^{b}, \ldots$ form a group $C_{n !}$ simply isomorphic with the symmetric permutation group $G_{n !}$.

The ratios $s_{i}$ are given in terms of the ratios $r_{i}$ of E. H. Moore by the formula

$$
r_{i j k l}=\left[r_{i}, r_{j}, r_{k}, r_{l}\right], \quad\left(r_{1}=\infty, r_{2}=0, r_{3}=1\right) .
$$

The $r_{i}$ can be expressed in terms of the $s_{i}$ by means of (3) and (3a). Thus the group $C_{n !}$ is equivalent to that of E. H. Moore under the transformation

$$
s_{i}=\left[r_{i-1}, r_{i-2}, r_{i}, r_{i-3}\right], \quad(i=4,5, \cdots, n) .
$$

3. Convergence Criteria for Continued Fractions. Let $C_{m !}$ be the subgroup of $C_{2 m \text { ! }}$ corresponding to permutations of the form

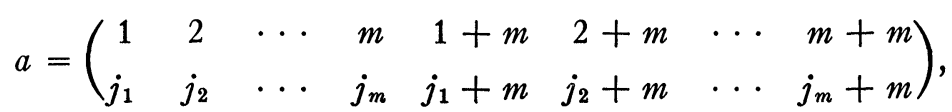

in which $j_{1}, j_{2}, \cdots, j_{m}$ is a permutation of $1,2, \cdots, m$. It is plain that $f_{4}{ }^{a}, f_{5}{ }^{a}, \cdots, f_{m}{ }^{a}$ are functions of $s_{4}, s_{5}, \cdots, s_{m}$ alone, while $f_{m+4}^{a}, f_{m+5}^{a}, \cdots, f_{2 m}^{a}$ are functions of $s_{m+4}, s_{m+5}, \cdots, s_{2 m}$ alone. The remaining three functions depend in general upon $s_{4}, s_{5}, \cdots, s_{2 m}$.

Let $k \geqq 0, m \geqq 3, z_{i+1}=A_{k m+i} / B_{k m+i},(i=0,1, \cdots, 2 m-1)$. Then $s_{i}=x_{k m+i-1},(i=4,5, \cdots, 2 m)$. We have

$x_{k m+i-1}^{a}=f_{i}^{a}\left(x_{k m+3}, x_{k m+4}, \cdots, x_{(k+2) m-1}\right), \quad(i=4,5, \cdots, 2 m)$, with the exceptional values*

* Perron, loc. cit., p. 198. 


$$
\begin{aligned}
& x_{0}{ }^{a}=\frac{A_{j_{1}-1}}{B_{j_{1}-1}}, \quad x_{1}{ }^{a}=\frac{A_{j_{1}-1}}{B_{j_{1}-1}}-\frac{A_{j_{2}-1}}{B_{j_{2}-1}}, \\
& x_{2}{ }^{a}=\left(\frac{A_{j_{8}-1}}{B_{j_{3}-1}}-\frac{A_{j_{2}-1}}{B_{j_{2}-1}}\right) /\left(\frac{A_{j_{2}-1}}{B_{j_{2}-1}}-\frac{A_{j_{1}-1}}{B_{j_{1}-1}}\right) .
\end{aligned}
$$

Then the continued fraction (with real or complex elements)

$$
\xi_{a}=x_{0}{ }^{a}-\frac{x_{1}^{a}}{1}-\frac{x_{2}{ }^{a}}{1}-\frac{x_{3}{ }^{a}}{1}-\ldots
$$

has the same convergents as $\xi$, but in a different order. Consequently, if $W_{a}$ is a region in $m$-space such that when the points

$$
\left(x_{k m}, x_{k m+1}, \cdots, x_{(k+1) m-1}\right), \quad(k=0,1,2, \cdots),
$$

range over $W_{a}$ we shall always have*

$$
\left|x_{n}{ }^{a}\right| \leqq \frac{1}{4}, \quad(n=2,3,4, \cdots),
$$

then $\xi$ converges. In this manner every transformation of the group $C_{m}$ ! gives a convergence theorem for $\xi$.

4. The Groups $C_{120}$ and $C_{120}$. As an illustration, $\dagger$ we shall consider the groups $C_{5 !}$ and $C_{51}$. The group $C_{120}$ is generated by the four transformations

$$
K \sim(34), \quad L \sim(23)(45), \quad M \sim(45), \quad T \sim(12) .
$$

The three transformations $K, L, M$ generate by themselves a subgroup $C_{24}$ of the main group $C_{120} ; T$ will extend $C_{24}$ to the main group.

By (3) and (3a) the transformations $K, L, M, T$ are found to be as follows:

$$
\begin{array}{ll}
K: x^{\prime}=\frac{x}{x-1}, y^{\prime}=\frac{1}{y} ; & L: x^{\prime}=\frac{1-y}{x}, y^{\prime}=y ; \\
M: x^{\prime}=\frac{x}{1-y}, y^{\prime}=\frac{y}{y-1} ; & T: x^{\prime}=\frac{x}{x-1}, y^{\prime}=\frac{y}{1-x},
\end{array}
$$

where, to avoid subscripts, we have put $s_{4}=x, s_{5}=y$.

* Perron, loc. cit., p. 259.

$\dagger$ The details of this illustration were worked out by Miss Lozelle Thomas. 
In the $x y$-plane the geometrical configuration for the group $C_{24}$ is as follows. The curves
1. $x^{2}=1-y$,
2. $y=0$,
3. $x=0$,
4. $y=(x-1)^{2}$,
5. $x=1$,
6. $y=1$,
7. $x+y=1$,
8. $x^{2}+2 x y-2 x-y+1=0$,

divide the plane into 24 regions. Take the fundamental region $I$ to be the region in the second quadrant bounded by the curves 1,2 and 3 . The other regions in the second quadrant are then: $L$ bounded by $1,2,6 ; L K$ bounded by 6,$8 ; L K L$ bounded by 7,$8 ; M K L$ bounded by 4,7 ; and $M K$ bounded by 3,4 .

In the first quadrant the regions are: $K$ bounded by 3,8 ; $K L$ bounded by $5,6,8 ; K M K L$ bounded by $4,5,6$, extending to infinity; $M L K$ bounded by 4,$6 ; M L K M$ bounded by 2, 4, 6; $M L K L M$ bounded by $4,5,6 ; K L K$ bounded by $1,5,6 ; L K L K$ bounded by 1,$7 ; M K L M$ bounded by 4,7 ; and $K M K$ bounded by $2,3,4$.

In the third quadrant the regions are: $L M$ bounded by 1,2 ; and $M$ bounded by $1,2,3$.

In the fourth quadrant the regions are: $K M$ bounded by 2,3 , $8 ; K L M$ bounded by 5,$8 ; M L K L K$ bounded by 1,$5 ; M K L K$ bounded by 1,7 ; LKL $M$ bounded by 7, 8; and $L K M$ bounded by 2,8 .

The generators of $C_{120}$ correspond to the permutations (34) (89), $(23)(45)(78)(9,10),(45)(9,10)$, and (12) (67). If we denote them by $K^{\prime}, L^{\prime}, M^{\prime}, T^{\prime}$, respectively, then these transformations are found to be

$$
\begin{aligned}
& x^{\prime}=\frac{x}{x-1}, \quad y^{\prime}=\frac{1}{y}, \quad \bar{x}^{\prime}=\frac{\bar{x}}{\bar{x}-1}, \quad \bar{y}^{\prime}=\frac{1}{\bar{y}}, \\
& K^{\prime} \text { : } \\
& u^{\prime}=\frac{u}{u-1}, \quad v^{\prime}=\frac{v}{1-w}, \quad w^{\prime}=\frac{w}{1-\bar{x}} ; \\
& x^{\prime}=\frac{1-y}{x}, \quad y^{\prime}=y, \quad \bar{x}^{\prime}=\frac{1-\bar{y}}{\bar{x}}, \quad \bar{y}^{\prime}=\bar{y}, \\
& L^{\prime} \text { : } \\
& u^{\prime}=\frac{1-y}{u}, \quad v^{\prime}=\frac{v}{v+w-1}, \quad w^{\prime}=\frac{w}{v+w-1} ;
\end{aligned}
$$




$$
x^{\prime}=\frac{x}{1-y}, \quad y^{\prime}=\frac{y}{y-1}, \quad \bar{x}^{\prime}=\frac{\bar{x}}{1-\bar{y}}, \quad \bar{y}^{\prime}=\frac{\bar{y}}{\bar{y}-1},
$$

$$
u^{\prime}=\frac{1}{u}, \quad v^{\prime}=\frac{v}{v-1}, \quad w^{\prime}=\frac{w}{1-v} ;
$$

$$
x^{\prime}=\frac{x}{x-1}, \quad y^{\prime}=\frac{y}{1-x}, \quad \bar{x}^{\prime}=\frac{\bar{x}}{\bar{x}-1}, \quad \bar{y}^{\prime}=\frac{\bar{y}}{1-\bar{x}},
$$

$T^{\prime}$ :

$$
u^{\prime}=\frac{u}{1-v}, \quad v^{\prime}=\frac{v}{v-1}, \quad w^{\prime}=\frac{1}{w},
$$

where for simplicity we have put $x=x_{5 m+3}, y=x_{5 m+4}, u=x_{5 m+5}$, $v=x_{5 m+6}, w=x_{5 m+7}, \bar{x}=x_{5 m+8}, \bar{y}=x_{5 m+9}$.

Each of these transformations yields a convergence theorem for the continued fraction $\xi$ in accordance with the remarks in $\S 3$. For example, $K^{\prime}$ gives the following theorem.

The continued fraction $\xi$ converges if the following inequalities hold:

$$
\begin{array}{r}
\left|\frac{x_{2}}{1-x_{3}}\right| \leqq \frac{1}{4} ; \quad\left|\frac{x_{5 n+3}}{x_{5 n+3}-1}\right| \leqq \frac{1}{4}, \quad\left|\frac{1}{x_{5 n+4}}\right| \leqq \frac{1}{4}, \\
(n=0,1,2, \cdots), \\
\left|\frac{x_{5 n}}{x_{5 n}-1}\right| \leqq \frac{1}{4},\left|\frac{x_{5 n+1}}{1-x_{5 n+2}}\right| \leqq \frac{1}{4}, \quad\left|\frac{x_{5 n+2}}{1-x_{5 n+3}}\right| \leqq \frac{1}{4}, \\
(n=1,2,3, \cdots),
\end{array}
$$

where $x_{1}, x_{2}, x_{3}, \cdots$ are real or complex numbers.

NORTHWESTERN UNIVERSITY 